\section{Caries dental en niños pre-escolares con síndrome Down}

Quijano GM, Díaz-Pizán, ME. Caries dental en niños pre-escolares con síndrome de Down. Rev Estomatol Herediana 2005; 15(2): Rev Estomatol Herediana 2005; 15 (2) : 128 - 132.

\section{RESUMEN}

El objetivo del presente estudio fue comparar la experiencia y prevalencia de caries dental en niños pre-escolares con diagnóstico de síndrome Down y normales. El estudio fue observacional descriptivo de corte transversal. Se examinaron 42 niños con síndrome Down y 50 niños normales de 3 a 6 años de edad, quienes cursaban estudios en dos Centros Educativos ubicados en el Cono Norte de Lima Metropolitana. Se realizó el análisis univariado de la variable caries dental observándose frecuencias absolutas y relativas en los dos grupos de estudio, según género y edad; se compararon directamente los valores utilizando el análisis divariado. Para el diagnóstico de experiencia de caries dental se utilizó el índice ceod (OMS). Los niños Down presentaron mayor experiencia de caries dental $(\operatorname{ceod}=4,36)$ que los niños normales $(\operatorname{ceod}=1,76)$. Los niños normales presentaron menor prevalencia de caries dental $(42 \%)$ a diferencia de los niños Down quienes estuvieron afectados en casi el $70 \%$.

Palabras clave: SÍNDROME DE DOWN / CARIES DENTAL.

\section{Dental caries in pre-school children with Down Syndrome}

\section{ABSTRACT}

The purpose of this study was to compare the experience and prevalence of dental caries in preschool children diagnosed with Down and normal children. The study was cross-sectional observational descriptive. 42 children with Down Syndrome and 50 normla children, ages to 6 , were examined who were enrolled in two kindergartens in the North Cone of Metropolitan Lima. A univaried analysis of the variable dental caries was performed, observing absolute and relative frequencies in the two groups studied, by gender and age; the values were directly compared using divaried analysis. The diagnosis of dental caries was done using the ceod (WHO) index. The Down children presented a greater average of experience of dental caries $($ ceod $=4.36)$ than normal children (ceod=1.76). Normal children presented lower prevalence of dental caries $(42 \%)$ as compared to Down children who were affected in almost $70 \%$ of the cases.

Keywords: DOWN SYNDROME / DENTAL CARIES.

\section{Gloria María Quijano Villavicencio' María Elena Díaz Pizán ${ }^{2}$}

${ }^{1}$ Residente del Programa de Odontología Pediátrica Postgrado.

2Docente del Departamento Académico de Estomatología del Niño y del Adolescente.

Facultad de Estomatología. Universidad Peruana Cayetano Heredia.

\title{
Correspondencia
}

Gloria Quijano Villavicencio,

Pasaje Juan Parra del Riego 254, Lima - 41, Perú Teléfono: 5337418

e-mail: glomarixx@ latinmail.com

\section{Introducción}

La primera descripción de un niño que tenía probablemente síndrome Down (SD) fue proporcionada por Esquirol en 1838. Ocho años más tarde, según describió un paciente con características sugeridas de una anomalía, que luego se convirtió en el conocido SD. En 1866, Juan L. Down publicó un artículo que describía algunas de las características de este síndrome que hoy lleva su nombre. Recién en 1959, Lejeune et al. concluyeron que los pacientes con SD tenían 47 cromosomas en lugar de 46 (1).

Al síndrome de Down se le conoce también como trisomía 21, trisomía $\mathrm{G}$, trisomía G-1. Se presenta como consecuencia de un reparto anormal de cromosomas, en donde las células llevan en su núcleo un cromosoma "extra", es decir 47 cromosomas en vez de 46 (1-8). Existen tres tipos de trisomía 21: Regular o parcial la cual se encuentra en el $90 \%$ a $95 \%$ de los casos; mosaicismo donde sólo una proporción del total de células tienen un cromosoma extra 21; mientras que la otra porción de células son normales, y traslocación que se presenta de $1 \%$ a $4 \%$ de los casos, en donde una parte del cromosoma del par 21 se encuentra "pegado" con otro cromosomas diferente (2-7).

El síndrome Down se presenta en 1/ 700 nacimientos de la población general, sin distinción de raza o de status cultural y socio-económico $(5,7)$.

Los pacientes con síndrome Down presentan determinadas características generales $(2,8)$, y múltiples anomalías congénitas (3-10), entre ellas malformaciones cardiacas aproximadamente en el $40 \%$ de ellos (3,8-10), así como características estomatológicas particulares (26,8,11-30).

Con respecto a caries dental en sujetos con SD, una minuciosa revisión de la literatura muestra dos posiciones diferentes:

a) Por un lado, algunos autores sostienen que estos pacientes presentan un menor riesgo de caries dental (11,
$12,14,21)$, debido a un $\mathrm{pH}$ salival alcalino, concentraciones más altas de bicarbonato y proteínas en saliva $(6,8,51)$; menor recuento de streptococcus mutans $(6,8,16,17,21)$ ya que un menor número de lesiones cariosas albergarían también menor cantidad de estos microorganismos; hipodoncia $(6,8,13)$ que llevaría a una dentición mas espaciada; erupción retardada de piezas dentarias $(6,8,10$, 12); fosas y fisuras superficiales en molares y premolares disminuyendo las zonas retentivas $(6,8)$; elevada concentración de IgA específicas para streptococcus mutans, lo cual inhibiría la adherencia bacteriana de estos microorganismos a los tejidos duros en la cavidad bucal (32). Se ha señalado una correlación positiva entre menor severidad de caries dental y cantidad de anticuerpos IgM, específicos a streptococcus mutans, sin embargo aún no está claro si este anticuerpo cumpliría un papel protector o responsable en la reducción de 
caries dental en este grupo de niños (33).

b) Mientras que otros investigadores afirman que estos pacientes presentan una mayor prevalencia de caries dental (22-25) y las causas serían: menor velocidad de flujo salival $(34,35)$, malos hábitos de higiene bucal (36) debido a una deficiente motricidad fina y a la poca orientación de los padres o tutores en este aspecto; dietas cariogénicas ya que los padres complacen frecuentemente a sus niños con dulces $(6,8,24)$, y el uso de mayor cantidad de jarabes debido a mayor susceptibilidad a infecciones $(6,8)$. Otras de las causas de una mayor cantidad de lesiones de caries dental en los niños Down sería que estos niños, a diferencia de los normales, dejan el pecho o biberón a una edad mayor, lo cual los predispone a mayor riesgo $(6,8,24)$. Así mismo, en la infancia los alimentos sólidos por lo general son ofrecidos a los niños Down más tardíamente $(6,8,24)$, y el destete ocurre por lo general después del año y medio (37). A todo ello se suman las pocas medidas preventivas en salud bucal, el escaso consumo de suplementos fluorados $(8,37)$, la incapacidad física e intelectual que no les permite una adecuada higiene bucal $(6,8)$, y la dificultad de encontrar servicios médico-odontológicos especializados $(6$, 8,38).

El propósito del presente estudio fue comparar prevalencia y experiencia de caries dental en dos grupos de niños pre-escolares uno con SD y el otro sin el síndrome, nuestra preocupación surgió debido a la controversia encontrada en la literatura y a la ausencia de investigaciones sobre este tema en niños con dentición decidua.

\section{Materiales y método}

El diseño del presente estudio fue de tipo observacional, descriptivo de corte transversal. La población estuvo conformada por niños pre-escolares de Centros Educativos del Cono Norte de Lima Metropolitana. Se evaluaron 42 niños con síndrome de Down pertene- cientes al C.E.E. Manuel Duato y 50 niños normales del C.E.I. 349 Palao - SMP, todos ellos con dentición decidua completa y pertenecientes a un nivel socioeconómico medio.

El número representativo para cada grupo de estudio se estimó considerando el estudio realizado por Iglesias (24). El muestreo fue realizado por conveniencia, considerando: tiempo, recursos para la investigación y accesibilidad de los centros educativos.

El proceso de calibración se dividió en dos fases :

1. En la primera fase se realizó la unificación criterios, procedimientos y aplicación del indicador de confiabilidad y concordancia (prueba Kappa de Cohen), obteniéndose los siguientes resultados: confiabilidad inter-examinador de la experiencia de caries dental 0,90 , y variabilidad intra-examinador para caries dental 0,77.

2. En la segunda fase se realizó la prueba piloto, que tuvo como objetivo probar la ficha clínica elaborada para fines del estudio y realizar el examen y diagnóstico de los niños en las mismas condiciones en las que se realizaría la investigación.

Se solicitaron las autorizaciones respectivas a los Centros Educativos y a los padres de los niños a fin que sus hijos formaran parte del estudio.

Las evaluaciones clínicas se realizaron entre las 9:00 am y 10:30 am en un ambiente iluminado con luz natural, el niño fue ubicado en una silla con respaldar alto, mientras el examinador, se hallaba de pie detrás de la silla. Para ello se contó con el apoyo de dos asis- tentas dentales entrenadas con el uso de la ficha clínica. Después del cepillado previo, se procedió a observar las lesiones de caries dental por cuadrantes utilizando el índice ceod (OMS) (39), para ello se utilizaron espejos bucales, bombilla de aire para secar las superficie dental y un explorador curvo.

Se realizó un análisis univariado para obtener frecuencias absolutas y relativas de la variable caries dental en niños normales y con Síndrome Down según género y edad, un análisis bivariado para comparar directamente los niveles de caries dental en los niños normales y con Síndrome Down de cada grupo por sexo y edad. Los resultados sólo permitieron expresar valores similares o distintos, debido a la naturaleza de conformación de los grupos (por conveniencia).

\section{Resultados}

De los 92 niños examinados 50 fueron normales (22 niñas y 28 niños) y 42 con diagnóstico de síndrome Down (16 niñas y 26 niños).

El ceod en niños normales fue igual a 1,76 y en los niños con síndrome Down de 4,23 dientes, existiendo una diferencia de medias de 2,6 dientes. El análisis por componentes muestra que la media del componente cariado en los niños Down fue de 4,05 a diferencia de los niños normales que presentaron valores menores $(1,52)$; la media del componente extraído fue mayor en los niños Down $(0,26)$, comparado con los normales $(0,02)$. Al analizar el componente obturado observamos lo contrario, los niños Down registraron menores valores $(0,05)$ que los normales (Tabla 1).

Tabla 1. Caries dental en niños normales y con síndrome de Down.

\begin{tabular}{lccccc}
\hline caries & \multicolumn{2}{c}{ niños normales } & \multicolumn{2}{c}{ niños con Down } & diferencia \\
(indicador) & media & DE & media & DE & de medias \\
\hline nddp & 19,98 & 0,14 & 19,74 & 1,08 & 0,24 \\
ndds & 18,22 & 2,70 & 15,64 & 4,23 & 2,58 \\
C & 1,52 & 2,34 & 4,05 & 3,79 & $-2,53$ \\
E & 0,02 & 0,14 & 0,26 & 1,08 & $-0,24$ \\
O & 0,18 & 0,69 & 0,05 & 0,22 & 0,13 \\
Ceod & 1,76 & 2,65 & 4,36 & 4,23 & $-2,60$ \\
\hline
\end{tabular}

nddp $=$ número de dientes deciduos presentes. ndds $=$ número de dientes deciduos sanos. $\mathrm{SD}=$ Síndrome Down. ceod= índice de experiencia de caries dental en dientes deciduos. D.E = Desviación estándar. $\mathrm{c}=$ cariado. $\mathrm{e}=$ extraído. $\mathrm{o}=\mathrm{obturado}$. 
En el grupo de niños normales el promedio ceod fue 1,68 dientes para el sexo femenino y 1,75 para el masculino, encontrándose una diferencia mínima entre ambos valores $(0,02)$. Del mismo modo, el promedio ceod para el grupo con síndrome Down fue 4,44 dientes para las niñas y 4,31 dientes para los niños, encontrándose también una diferencia estrecha entre ambos valores $(0,13)$ (Tabla 2).

Tanto en el grupo de niños normales como en los niños con SD el promedio ceod se incrementó paulatinamente según la edad de los niños evaluados, disminuyendo ligeramente a la edad de 6 años (Tabla 3, Fig. 1).

Los niños normales presentaron menor prevalencia de caries dental (42\%) a diferencia de los niños Down que obtuvieron casi el 70\% (Fig. 2).

\section{Discusión}

En lo referente a experiencia de caries en niños con síndrome Down los resultados del presente estudio fueron similares a los de Hernández et al. (12) quienes en una muestra de niños mexicanos del estado de Yucatán de 4 a 6 años de edad, encontraron un ceod de 4,16 . Sin embargo, valores menores a los nuestros fueron obtenidos por otros investigadores sudamericanos en niños con este síndrome $(14,19,22)$.

Los resultados del presente estudio, así como también los de Hernández et
Tabla 2. Caries dental en niños normales y con síndrome de Down según género.

\begin{tabular}{lllllllll}
\hline & \multicolumn{3}{c}{ femenino } & \multicolumn{3}{c}{ masculino } & \multicolumn{2}{c}{ total } \\
grupo & indicador & media & DE & & media & DE & media & DE \\
\hline \multirow{4}{*}{ normal } & nddp & 19,95 & 0,21 & 20,00 & 0,00 & 19,98 & 0,14 \\
& ndds & 18,18 & 2,82 & 18,25 & 2,65 & 18,22 & 2,70 \\
& C & 1,41 & 2,24 & 1,61 & 2,45 & 1,52 & 2,34 \\
& E & 0,05 & 0,21 & 0,00 & 0,00 & 0,02 & 0,14 \\
& O & 0,23 & 0,61 & 0,14 & 0,76 & 0,18 & 0,69 \\
& ceod & 1,68 & 2,61 & 1,75 & 2,65 & 1,76 & 2,65 \\
\hline \multirow{4}{*}{ SD } & nddp & 19,69 & 1,25 & 19,77 & 0,99 & 19,74 & 1,08 \\
& ndds & 15,56 & 4,26 & 15,69 & 4,30 & 15,64 & 4,23 \\
& C & 4,06 & 3,99 & 4,04 & 3,75 & 4,05 & 3,79 \\
& E & 0,31 & 1,25 & 0,23 & 0,99 & 0,26 & 1,08 \\
& O & 0,06 & 0,25 & 0,04 & 0,20 & 0,05 & 0,22 \\
& ceod & 4,44 & 4,26 & 4,3 & 4,30 & 4,36 & 4,23 \\
\hline
\end{tabular}

nddp= número de dientes deciduos presentes. ndds = número de dientes deciduos sanos. $\mathrm{SD}=$ Síndrome Down. ceod= índice de experiencia de caries dental en dientes deciduos. D.E $=$ Desviación estándar. $\mathrm{c}=$ cariado. $\mathrm{F}=$ Femenino. $\mathrm{e}=$ extraído. $\mathrm{M}=$ Masculino. $\mathrm{o}=$ obturado.

Tabla 3. Experiencia de caries dental en niños normales y con síndrome down según edad.

\begin{tabular}{llrrrrrrrrr}
\hline & indicador & \multicolumn{3}{c}{03 años } & \multicolumn{2}{c}{04 años } & \multicolumn{3}{c}{05 años } & \multicolumn{2}{c}{06 años } \\
\hline grupo & & media & DE & \multicolumn{1}{c}{ media } & DE & media & DE & media & DE \\
normal & nddp & 20,00 & 0,00 & 19,90 & 0,32 & 20,00 & 0,00 & 20,00 & 0,00 \\
& ndds & 18,94 & 2,25 & 17,10 & 3,41 & 17,15 & 3,05 & 19,50 & 0,85 \\
& C & 1,06 & 2,25 & 1,60 & 1,90 & 2,85 & 3,05 & 0,50 & 0,85 \\
& E & 0,00 & 0,00 & 0,10 & 0,32 & 0,00 & 0,00 & 0,00 & 0,00 \\
& O & 0,00 & 0,00 & 0,90 & 1,37 & 0,00 & 0,00 & 0,00 & 0,00 \\
& ceod & 1,06 & 2,25 & 2,60 & 3,10 & 2,85 & 3,05 & 0,50 & 0,85 \\
\hline SD & nddp & 20,00 & 0,00 & 19,58 & 1,44 & 19,54 & 1,39 & 20,00 & 0,00 \\
& ndds & 17,33 & 3,04 & 16,00 & 4,79 & 14,23 & 4,36 & 15,50 & 4,24 \\
& C & 2,67 & 3,04 & 3,58 & 3,75 & 5,15 & 4,06 & 4,50 & 4,24 \\
& E & 0,00 & 0,00 & 0,42 & 1,44 & 0,46 & 1,39 & 0,00 & 0,00 \\
& O & 0,00 & 0,00 & 0,00 & 0,00 & 0,15 & 0,38 & 0,00 & 0,00 \\
& ceod & 2,67 & 3,04 & 4,00 & 4,79 & 5,77 & 4,36 & 4,50 & 4,24 \\
\hline
\end{tabular}

nddp $=$ número de dientes deciduos presentes. ndds $=$ número de dientes deciduos sanos. $\mathrm{SD}=$ Síndrome Down. ceod= índice de experiencia de caries dental en dientes deciduos. D.E $=$ Desviación estándar. $\mathrm{c}=$ cariado. $\mathrm{e}=$ extraído. $\mathrm{o}=$ obturado

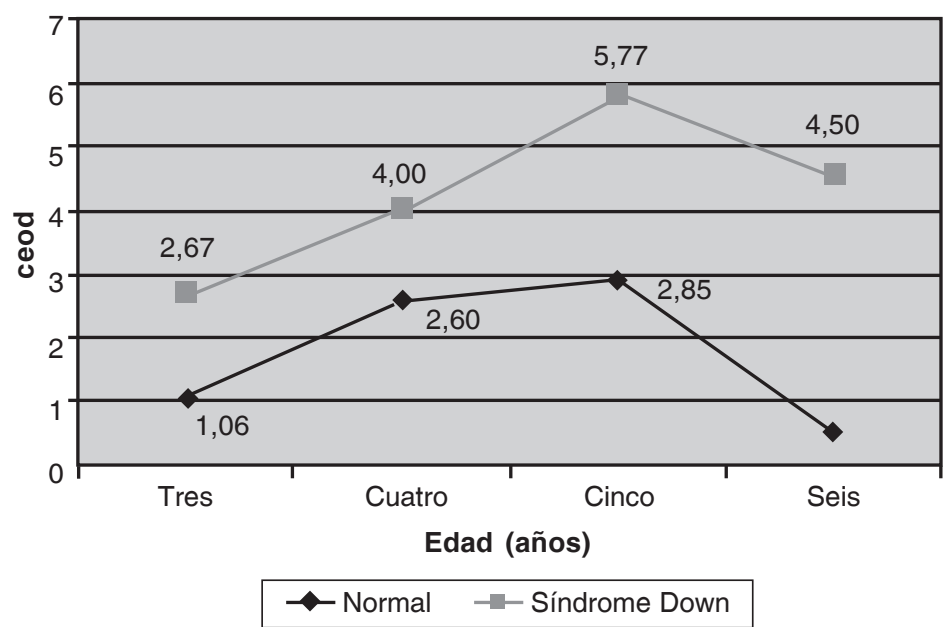

Figura 1. Experiencia de caries dental en niños normales y con síndrome down según edad.

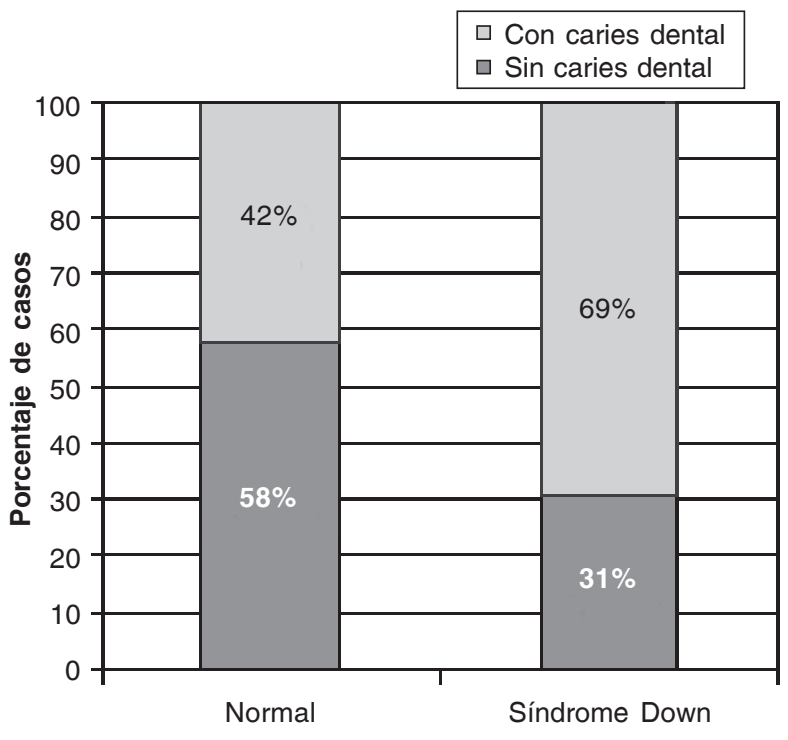

Figura 2. Prevalencia de caries dental y porcentaje (\%) libres de caries dental en niños normales y con síndrome down. 
al. (12), y Menacho (14), todos ellos en niños Down con dentición decidua, están en el rango de caries moderada según los lineamientos de la OMS (40). Mientras que los estudios de Bianchi y Jaramillo (22) dentro del rango bajo. Una explicación a ello podría ser que estos pacientes con síndrome Down son menos susceptibles a caries dental por poseer un $\mathrm{pH}$ salival más alto, recuento de Streptococcus mutans en cantidades menores y mayor concentración de Ig $\mathrm{A}(6,8,14,16,17,32,33)$. Algunos autores señalan también que la hipodoncia y la erupción retardada $(6,8,13)$, serían factores protectores contra la caries dental; sin embargo, en nuestro estudio no encontramos ningún paciente Down con hipodoncia o erupción retardada, descartando estos elementos como factores de protección.

Una de las grandes limitaciones encontradas en el presente estudio, fue el limitado número de investigaciones en niños de estas edades a nivel mundial. La gran mayoría de autores incluyen en la misma muestra a niños, adolescentes $\mathrm{y}$ adultos; lo cual impide un análisis de la experiencia de caries dental por grupos de edad y específicamente en preescolares.

El ceod encontrado en el grupo de niños normales fue de 1,76 que según la OMS (41) estaría en el rango bajo. Últimos estudios (41-45) que valoraron el ceod en poblaciones pre-escolares, revelan valores bastante mayores a los encontrados en el nuestro. Podemos decir pues que nuestros resultados fueron menores probablemente por ser una población que está dentro de la jurisdicción de la Clínica Dental de la Universidad Peruana Cayetano Heredia, lo que los hace de alguna manera un grupo privilegiado dentro de la población general, ya que se benefician de los diferentes programas de prevención, promoción y tratamiento de la cavidad bucal.

Al comparar los valores de experiencia de caries dental entre ambos grupos encontramos que los niños Down duplican los valores de caries dental. Situación similar fue hallada por Bianchi y Jaramillo (22) quienes encontraron un ceod de 2,4 para pacientes Down y 0,8 para pacientes sin el síndrome.
El porcentaje de los sujetos con caries dental en ambos grupos podemos mencionar que el $69 \%$ de los niños Down presentaron alguna lesión cariosa a diferencia del $42 \%$ que presentaron los niños normales.

El análisis de la experiencia de caries dental por edad, en ambos grupos revela, que el ceod aumentó conforme aumentó la edad de los grupos examinados, observado esto en los niños de 3,4 y 5 años para disminuir a los 6 años de edad. Esto concuerda con los resultados de Rivera (46) quien señala que la severidad de caries en dentición decidua, disminuye con la edad, debido probablemente al proceso de exfoliación de las piezas deciduas. En el caso de los niños Down, en todos los grupos de edad, los valores ceod fueron significativamente mayores.

Lamentablemente la evaluación de los niños fue realizada en el mismo centro educativo donde no se contó con las comodidades de una unidad dental que permitiera la correcta evaluación de las lesiones de mancha blanca (estadio inicial de la enfermedad). Una evaluación que hubiera incluido este estadío, probablemente nos hubiera dado valores ceod mayores. Algunos investigadores $(45,47)$ al respecto indican que una evaluación de caries dental a partir de lesiones cavitadas produce un subregistro de la enfermedad.

\section{Conclusiones}

- Los niños Down presentaron mayor promedio de experiencia de caries dental que los niños normales.

- El ceod fue mayor en los pacientes con Síndrome Down, tanto en las niñas como en niños, en los diferentes grupos de edades.

- Los niños normales presentaron menor prevalencia de caries dental a diferencia de los niños Down.

\section{Referencias bibliograficas}

1. López M, López R, Perez G, Borges A, Valdespino L. Reseña histórica del Síndrome de Down. Rev ADM, 2000; 5:193-199.

2. Cabrera A. Caracteres clínicos del recién nacido con síndrome de Down. Diagnóstico 1984; 13(3):97-100.
3. Gallardo B. Síndrome de Down: un reto para el pediatra de hoy. Paediatrica 2000; 3(2):22-27.

4. Jasso L .El niño Down mitos y realidades. México: Editorial El Manual Moderno; 1991.

5. Quiroga M. Síndrome de Down guía para profesionales y padres. Lima:Editorial Instituto de Genética UPCH; 1992.

6. Desaii S, Fayetteville B. Down syndrome. Oral Surg Oral Pathol Oral Radiol Endond 1997; 84(3):279-85.

7. Saenz R. Cuidado primario de infantes y de niños jóvenes con síndrome de Down. American Fam Physic 1999; 59:381-386.

8. Fiske J, Shafik H. Down's syndrome and oral care. SADJ 2001; 56(9): 407415.

9. Castillo W. Inmunología en el síndrome de Down. Diagnóstico 1984; 13(5):165-168.

10. Van Dyke M. Medical \& Surgical, Care for children down's Syndrome, a guide for parent. Nueva York: Editorial Woodbine House; 2000.

11. Pirela de Manzano M, Salazar C, Manzano M.. Patología bucal prevalente en niños excepcionales. Acta Odontol Ven 1990; 37(3): 193-197.

12. Hernandez J, Tello T, Ochoa G. Alteraciones bucales en niños con Síndrome de Down en el estado de Yucatán. Rev ADM 1998; (2): 91-96.

13. Goes A, Freitas C, Cury M. Prevalence of numeric anomalies in the permanent dentition of patients with Down syndrome. Spec Care Dent 2001;21 (2): 75-78.

14. Menacho A. Perfil de salud oral en el Síndrome de Down, Prevalencia de la enfermedad periodontal y caries dental y su correlación con higiene oral en individuos con Síndrome de Down en centros de Educación Especial en Lima. [Tesis de bachiller]. Lima-Perú: Universidad Peruana Cayetano Heredia; 1985.

15. Maclaurin E, Shaw T, Foster D. Dental caries and periodontal in children with Down's syndrome and other mentally handicapping conditions. J Pediatr dent 1985; 1:15-19.

16. Stabholz A, Mann J, Sela M, Schurr D, Steinberg D, Shapira J. Caries 
experience, periodontal treatment needs, salivary $\mathrm{pH}$, and streptococcus mutans counts in a preadolescent Down syndrome population. Spec Care Dentist 1991; 11(5):203-208.

17. Shapira J, Stabholz A, Mann J, Sela M, Schurr D, Steinberg D. Caries levels, streptococcus mutans counts, salivary $\mathrm{pH}$ and periodontal treatment needs of adults Down syndrome patients. Spec Care Dentist 1991; 11(6): 248-251.

18. Pinazo J, Vianna M, Lopez F. Carie dentaria e placa bacteriana em criamcas de 07 a 14 años portadores de síndrome de Down, matriculados em institucões publicas e privadas do Municipio de Salvador - Bahia. Rev Fac Odontol UFBA 1998; 17:15-24.

19. López R, López P, Borges A, Pares G. Caries dental en niños con trisomía 21. Bol Med Hosp. Infant Mex 2000; 57 (8): 438-443.

20. Leonelli de Moraes M, Silva M, Moraes C, Rocha C. Prevalencia de càrie pelo índice $\mathrm{CPO}-\mathrm{D}$ em portadores de síndrome de Down. Posgrad Rev Odontol 2002; 5 (2): 64-73.

21. Bisso F. Caries dental, pH salival y Niveles de streptococcus mutans en adolescentes con Síndrome de Down y adolescentes normales de la ciudad de Lima. [Tesis de bachiller]. Lima-Perú: Universidad Nacional Mayor de San Marcos; 2003.

22. Bianchi A, Jaramillo R. Investigación odontológica en personas con Síndrome de Down. RAOA 1991; 79(3):147-152.

23. Ulseth J, Hestens A, Stovner L, Storhaug K. Dental caries and periodontitis in persons with Down syndrome. Spec Care Dentist 1991; 11(2):71-73.

24. Iglesias N. Prevalencia de càrie dentaria em criancas portadoras de síndrome de Down na faixa etaria de 0 a 60 meses. JBP 2000; 3(12):147-157.

25. Shyama M, Mutawa S, Morris R, Sugathan T, Honkala E. Dental caries experience of disabled children and young adults in Kuwait. Community Dent Health 2001; 18:181-186.

26. Vittek J,Winik S, Winik A, Sioris C,
Tarangelo A, Chou M. Analysis of orthodontic anomalies in mentally retarded developmentally disabled (MRDD) persons. Spec Care Dentist 1994; 14(5):198-202.

27. Rodríguez C, Bratos E, Garcillan R, Rioboo R. Estudio epidemiológico en adultos discapacitados: Parte III: Maloclusión y otras anomalías dentarias. Avances en odontoestomatología 2000; 16(9):527-534.

28. Barr M, Dahllof G, Modéer T, Per E, Norhagen G. Periodontal conditions and salivary immunoglobulins in individuals with Down syndrome. J Periodontol 1998; 69 (10):1119-1123.

29. Lopez R, Borges A, Jímenes G, Maupome G. Oral hygiene, gingivitis and periodontitis in persons with Down syndrome. Spec Care Dentist 2002; 22 (6): 214-220.

30. Yasaki Y, Sumi Y, Miyasaki Y, Hamachi T, Nakata M. Periodontal management of an adolescent with Down's syndrome - a case report. Int J Pediatr Dent 2004; 14(2):127-135.

31. Winer R, Féller R. Composition of parotid and submandibular saliva and serum in Down's syndrome. J Dent Res 1972; 51:449-54.

32. Lee S, Know H, Song K, Choi Y. Dental caries and salivary inmunoglobulin A in Down Syndrome children. J Paediatr Child Health 2004; 40(9-10): 530-3.

33. Morinushi T, Lopatin D, Tanaka H. The Relationship between dental caries in the primary dentition and anti S. mutans serum antibodies in children with Down's Syndrome. J Clin Pediatr Dent 1995; 19(4):279-283.

34. Yarat A, Akyüz S, Koc L, Erdem H, Emekli N. Salivary sialic acid, protein, salivary flow rate, $\mathrm{pH}$, buffering capacity and caries indices in subjects with Down's Syndrome. J Dent 1999; 27:115-118.

35. Siquiera W, Nicolau J Stimulated whole saliva component in children with Down Syndrome. Spec Care Dentist 2002; 22(6):226-230.

36. Allison A, Lawrence H. A Paired comparison of dental care in Canadians with Down Syndrome and their siblings without Down syndrome.
Comunity Dent Oral Epidemiol 2004;32:99-106.

37. Randell D, Harth S, Seow W. Preventive dental health practices of non-institucionalized Down syndrome children: A controlled study. J Clin Pediatr Dent 1992; 16(3):225-229.

38. Allison P, Hennequin M, Faulks D. Dental care among individuals with Down syndrome in france. Spec Care Dentist 2000; 20(1):28-34.

39. OMS. Encuestas de salud bucal. Métodos básicos. 4ed. Suiza: Editorial OMS; 1997.

40. OMS. Encuestas de salud bucal. Métodos básicos. Suiza: Editorial OMS; 1984.

41. Weilg M. Estudio epidemiológico de salud oral en niños de 3-5 años, hijos de trabajadores del complejo metalúrgico de la Oroya. [Tesis de Bachiller]. Lima - Perú: Universidad Peruana Cayetano Heredia; 1987.

42. Anchiraico C. Estudio epidemiológico de salud oral en niños preescolares que asisten a los centros de educación inicial de Jauja. [Tesis de Bachiller]. Lima - Perú: Universidad Peruana Cayetano Heredia; 1991.

43. Shy W, Holm A, Yuen S. Dental caries prevalence and related factors in 5 years. Old children in Hong Kong. Pediatr Dent 1993; 15:106-109.

44. Wyne A, Darwish S, Adenubi J, Battata S, Khan N. The prevalence and pattern of nursing caries in Saudi Preschool Children. Int J Pediatr Dent. 2001; 11:361-364.

45. Gonzales M, Ruíz J, Fajardo M, Gomez A, Moreno C, Ochoa M, Rojas L. Comparison of the index with Nyvad's caries diagnostic criteria in 3 and 4 years-old Colombian Children. Pediart Dent 2003;25(2): 132-136.

46. Rivera S. Magnitud y severidad de caries dental en niños de 6 a 12 años de edad del Cuzco [Tesis Maestría]. Lima - Perú: Universidad Peruana Cayetano Heredia; 2004.

47. Nivad B, Machiulskiene V, Baelum $\mathrm{V}$. Reliability of a new caries diagnostic system differentiating between active and inactive caries lesions. Caries Res 1999; 33:252-260. 